\title{
Ventajas de la plastia de mielomeningocele y derivación ventrículo-peritoneal en único tiempo quirúrgico: Experiencia en 47 casos
}

\author{
José Luis Alcocer Maldonado, ${ }^{1}$ Ricardo Morales Montes, ${ }^{2}$ Luis Gerardo Domínguez Carrillo ${ }^{3}$
}

\section{Resumen}

Antecedentes: El mielomeningocele es el defecto del cierre del tubo neural más frecuente y compatible con la vida. El tratamiento iniciado desde el nacimiento mejora el pronóstico funcional y la calidad de vida del paciente, la principal complicación del mielomeningocele es la hidrocefalia que requiere el uso de derivación ventricular. Objetivo: Comparar las ventajas de cirugía de plastia del mielomeningocele y colocación de derivación ventrículo-peritoneal en un solo tiempo quirúrgico (grupo A) versus plastia clásica y derivación diferida (grupo B). Material y métodos: de 47 pacientes con mielomeningocele que cumplieron los criterios de inclusión, el tercero de cada tres recibió cirugía simultánea. Resultados: Los datos demográficos de edad, género, peso corporal, localización de la lesión y grados de hidrocefalia no mostraron diferencias estadísticamente significativas. Los tiempos anestésicos $(p=0.00001)$ y quirúrgicos fueron menores en el grupo $A(p=0.001)$ al igual que las pérdidas sanguíneas ( $p=0.003$ ) y los días de estancia hospitalaria $(p=0.01)$. El dato más relevante y significativo en el grupo A fue la disminución de complicaciones; de manera global la ventriculitis fue la complicación que surgió en ambos grupos. No se presentaron decesos a los 30 días en ninguno de los grupos. Conclusiones: La realización de plastia del mielomeningocele y derivación ventrículo-peritoneal en un solo tiempo muestra ventajas en todos los aspectos, principalmente en la reducción de complicaciones.

Palabras clave: Mielomeningocele y derivación ventrículoperitoneal simultáneas.

\begin{abstract}
Summary
Background: Myelomeningocele is a congenital neural tube defect compatible with life, an early treatment improves functional outcome and quality of life of patients. Hydrocephalus is the main complication, requiring insertion of a ventriculoperitoneal shunt. Objective: To compare these procedures in the same operative setting (group A) versus classic repair in a first time and ventriculoperitoneal shunt deferred to a second time (group B). Material and methods: Of 47 patients with myelomeningocele, 15 received simultaneous surgery. Results: Demographic data like: age, gender, body weight, lesion location and hydrocephalus degrees showed no statistically significant differences. Anesthetics time $(p=0.00001)$, surgical time ( $p$ $=0.001)$ as well blood loss $(p=0.003)$ and hospital stay $(p=$ 0.01 ); were lower in the group $A$, ventriculitis was the most frequent complication in both groups. No deaths occurred within 30 days in either group. Conclusions: In this series the early simultaneous performance of myelomeningocele repair and ventricularperitoneal shunt shows benefits in all aspects.
\end{abstract}

Key words: Simultaneous mielomeningocele repair and ventriculoperitoneal shunt.

\footnotetext{
${ }^{1}$ Neurocirujano. Jefe del Servicio de Neurocirugía del Hospital General de León. División de Cirugía del Hospital Ángeles León, León, Guanajuato, México.

2 Neurocirujano. Hospital de Alta Especialidad Núm. 48, Instituto Mexicano del Seguro Social. León, Guanajuato, México.

${ }^{3}$ Especialista en Medicina de Rehabilitación. Profesor de la Facultad de Medicina de León, Universidad de Guanajuato, México.
}

Correspondencia:

Dr. José Luis Alcocer Maldonado

Correo electrónico: alcocermjl@hotmail.com

Aceptado: 27-09-2016.

Este artículo puede ser consultado en versión completa en http://www.medigraphic.com/actamedica 


\section{INTRODUCCIÓN}

El mielomeningocele constituye el defecto del cierre del tubo neural más frecuente y compatible con la vida, ${ }^{1}$ asociado en su gran mayoría a deficiencia de ácido fólico. Sin embargo, a pesar de la contundente evidencia de la participación del ácido fólico en la prevención de los defectos del tubo neural existen casos denominados "no prevenibles por ácido fólico", en los que debe reconocerse la influencia de otros factores. En México afecta a 1.2:1,000 nacidos vivos, ${ }^{2,3}$ siendo discretamente más frecuente en el género femenino. En Estados Unidos el número de mielomeningoceles ha disminuido de manera considerable y las cirugías para su tratamiento se realizan principalmente en el grupo de pacientes hispanos, ${ }^{4}$ siendo una cirugía cada vez menos frecuente. Respecto a su fisiopatogenia las evidencias clínicas y experimentales sugieren que los defectos no son sólo la neurulación incompleta, sino que existe un trauma mecánico y químico del líquido amniótico que origina daño progresivo al tejido neural expuesto durante la gestación. ${ }^{5}$ La complicación primordial es hidrocefalia hasta en $95 \%$ de los $\operatorname{casos}^{6}$, lo que implica además de la plastia del mielomeningocele la necesidad de cirugía de derivación ventricular. Otras complicaciones $^{7}$ van desde vejiga neurogénica hasta la incapacidad para deambular, llevando al paciente a una discapacidad considerable, incluyendo elevados costos de manejo desde el nacimiento y numerosas cirugías a lo largo de la vida. ${ }^{8}$ El tratamiento iniciado desde el nacimiento mejora el pronóstico funcional y la calidad de vida. ${ }^{9} \mathrm{Al}$ respecto los progresos en el manejo quirúrgico postnatal han sido escasos, pues consisten en la cobertura de la médula espinal expuesta, prevenir la infección y tratar el hidrocéfalo con derivación (habitualmente) ventrículoperitoneal. ${ }^{10}$ El surgimiento de la reparación fetal ${ }^{1-11}$ del mielomeningocele ha mostrado que la cirugía intrauterina antes de la semana 26 de gestación $\mathrm{n}^{5-12}$ puede preservar la función neurológica, revirtiendo la herniación del tallo cerebral de la malformación de Chiari tipo II y evitando la necesidad de derivación ventricular; no obstante, esta cirugía tiene riesgos en la madre y aumenta la posibilidad de parto prematuro, además de las dificultades técnicas que implica, por lo que no puede practicarse de manera frecuente en nuestro medio. En México a nivel de las instituciones públicas del sector salud, la corrección quirúrgica del mielomeningocele se efectúa inicialmente en las primeras 72 horas y en cirugía posterior (generalmente entre seis y 12 días) se realiza la colocación de válvula de derivación ventrículo-peritoneal. No está generalizado el manejo de ambos procedimientos en un solo tiempo quirúrgico, aunque desde 1987 se menciona su utilidad y ventajas, existen aún algunas controversias.
Al tratar de disminuir la estancia hospitalaria, la morbilidad, el costo y el riesgo en este tipo de pacientes al ser sometidos a dos procedimientos anestésicos y quirúrgicos diferidos, se decidió, con base en la revisión de los estudios previos efectuados en otras latitudes, llevar a cabo ambas cirugías en un solo tiempo quirúrgico en uno de cada tres pacientes (muestra consecutiva y sistemática por conveniencia) con mielomeningocele que acudieran a la institución y cumplieran los criterios de inclusión, siendo el motivo de esta comunicación.

\section{MATERIAL Y MÉTODOS}

Con protocolo aprobado por los comités de ética e investigación de la institución del 1 de julio de 2011 al 31 de diciembre de 2014 se llevó a cabo estudio clínico, observacional, retrolectivo, retrospectivo, comparativo y transversal de pacientes con mielomeningocele sometidos a plastia del mismo, la muestra fue obtenida de manera no probabilística, consecutiva y sistemática, efectuándose la plastia del mielomeningocele y la derivación ventrículoperitoneal en un solo tiempo en el tercero de cada tres pacientes que cumplieran los siguientes criterios de inclusión: a) recién nacido con mielomeningocele; b) sin malformaciones congénitas asociadas; c) con hidrocefalia comprobada por ultrasonido y d) profilaxis con doble esquema antibiótico. Los criterios de exclusión fueron: otra malformación, insuficiencia respiratoria y pacientes que no concluyeron tratamiento. Se cuantificaron los datos demográficos de edad en días, género, peso, perímetro cefálico, localización del mielomeningocele y presencia de hidrocefalia. Se midieron tiempos anestésicos, tiempos quirúrgicos, cuantificación de sangrado transoperatorio, complicaciones transoperatorias y postoperatorias, los días de estancia hospitalaria y mortalidad a 30 días del procedimiento, así como seguimiento a un año. Los pacientes que cumplieron los criterios de inclusión conformaron la muestra, la cual fue dividida en dos grupos; el grupo A ( $\mathrm{n}$ =15) fue sometido a colocación de derivación ventrículo peritoneal en el mismo tiempo quirúrgico; en el grupo B $(n=32)$ la derivación ventrículo-peritoneal se realizó más tarde en un segundo tiempo, sumándose en este último grupo los tiempos anestésicos, quirúrgicos y de estancia hospitalaria de las dos cirugías efectuadas en diferentes tiempos. En todos los casos el mismo grupo neuroquirúrgico aplicó los procedimientos, ya fueran en uno o dos tiempos. Las válvulas colocadas a todos los pacientes fueron tipo Pudenz de mediana presión infantil marca Biomed. Se utilizaron promedios y desviación estándar, así como t de Student, chi cuadrada $\left(\chi^{2}\right)$ y prueba exacta de Fisher, considerando $p=0.05$ el valor estadísticamente significativo. Se dio seguimiento durante un año. 


\section{Breve descripción de la técnica quirúrgica}

Con el paciente en decúbito dorsal y la cabeza rotada a la derecha se practica antisepsia de cráneo, dorso y flanco abdominal. 1. Técnicamente se inicia con la colocación del sistema de derivación. 2 . Se practica la plastia del mielomeningocele. De manera inicial: a) se procede a realizar un trépano en la región parietal derecha, colocando el tambor del sistema de derivación; b) en la región del flanco derecho que corresponde al triángulo lumbar superior o espacio de Grynfeltt (el triángulo de Grynfeltt-Lesshaft ${ }^{13,14}$ está limitado medialmente por el músculo cuadrado lumbar, lateralmente por el músculo oblicuo interno del abdomen y superiormente por la duodécima costilla, el piso está formado por la fascia transversalis); c) se practica incisión de $1.5 \mathrm{~cm}$ visualizando al dorsal ancho, el cual se incide, se abre la cavidad peritoneal colocando la porción distal del catéter de derivación; d) la porción proximal del catéter se conduce vía subcutánea al cráneo hasta su conexión, se cierra por planos; e) tras cambio de guantes y material quirúrgico; f) se practica la plastia del mielomeningocele en la forma clásica.

\section{RESULTADOS}

Se revisaron 101 casos de pacientes con mielomeningocele, de los cuales 54 fueron excluidos por no cumplir los criterios de inclusión, 44 por tratamiento incompleto y 10 por presentar malformaciones congénitas asociadas. La muestra fue conformada por 47 pacientes, misma que fue dividida en dos grupos, el grupo A (un solo tiempo quirúrgico: plastia + derivación) formado por 15 casos (31.91\%) y el grupo B (dos tiempos quirúrgicos) por 32 casos (68.08\%). Correspondiendo a relación 1:2.

En relación con la edad, género y peso corporal no se encontró en ninguno de los grupos diferencia estadísticamente significativa, aunque el peso corporal fue más disperso en el grupo B. Respecto al perímetro cefálico los pacientes del grupo A mostraron un promedio más alto que los del grupo B con significancia estadística y el grado de hidrocefalia predominó en el nivel moderado en ambos grupos sin diferencia significativa al compararlos. La localización del mielomeningocele a nivel lumbar predominó en ambos grupos, seguida de la zona lumbosacra y por último la zona toracolumbar (Cuadro 1).

\begin{tabular}{|c|c|c|c|c|c|c|}
\hline Variable & Grupo A & $\%$ & Grupo B & $\%$ & $x$ & P. E. valor de $p$ \\
\hline $\mathrm{N}$ & 15 & 31.91 & 32 & 68.08 & & \\
\hline Edad/días X & 5.73 & & 5.87 & & 5 días & $t=0.017$ \\
\hline $\mathrm{DE} \pm$ & 2.091 & & 2.660 & & 6 días & $p=0.98 N S$ \\
\hline \multicolumn{7}{|l|}{ Género } \\
\hline Masculino & 8 & 53.3 & 13 & 40.6 & - & $\chi^{2}=0.2521$ \\
\hline Femenino & 7 & 46.7 & 19 & 59.4 & - & $p=0.61 N S$ \\
\hline \multicolumn{7}{|l|}{ Peso/gramos } \\
\hline Peso X & $3,015 \mathrm{~g}$ & & $3,100 \mathrm{~g}$ & & - & $t=0.31$ \\
\hline $\mathrm{DE} \pm$ & $458 \mathrm{~g}$ & & $480 \mathrm{~g}$ & & - & $p=0.75 \mathrm{NS}$ \\
\hline \multicolumn{7}{|l|}{ P. cefal./ cm } \\
\hline X 37.5 & 32.5 & & 36.4 & & - & $t=2.00$ \\
\hline $\mathrm{DE} \pm$ & 3.31 & & 3.52 & & - & $p=0.05 S$ \\
\hline \multicolumn{7}{|l|}{ Hidrocefalia } \\
\hline Mínima & 1 & 6.6 & 11 & 34.4 & - & \\
\hline Moderada & 10 & 66.7 & 17 & 53.1 & - & $\chi^{2}=4.6011$ \\
\hline Severa & 4 & 26.7 & 4 & 12.5 & - & $p=0.10 N S$ \\
\hline \multicolumn{7}{|l|}{ Loc. mielo } \\
\hline Lumbar & 7 & 46.7 & 12 & 37.6 & - & - \\
\hline Dorsal & 1 & 6.7 & 2 & 6.2 & - & - \\
\hline Lumbosacro & 4 & 26.6 & 8 & 25.0 & - & - \\
\hline Toracolumbar & 3 & 20.0 & 8 & 25.0 & - & - \\
\hline Sacro & 0 & 0 & 1 & 3.1 & - & - \\
\hline Cervico-dorsal & 0 & 0 & 1 & 3.1 & - & - \\
\hline
\end{tabular}


Respecto al tiempo anestésico, en el grupo A se requirió $X$ de 111 minutos, a diferencia del grupo $B$ que mostró $X$ de 166 minutos con una diferencia estadísticamente relevante (Cuadro 2).

Los tiempos quirúrgicos también mostraron diferencia estadísticamente significativa, pues el grupo $A$ mostró $X$ y DS de $81.7 \pm 19.5$ minutos, mientras que en el grupo $B$, el X y DE fue de $116 \pm 36.8$ minutos (Cuadro 2).

En relación con las complicaciones no se presentaron eventos durante el transoperatorio en ninguno de los grupos. Las cuantificaciones de pérdida sanguínea transoperatoria mostraron: $X$ y DE de $11.4 \mathrm{~mL} \pm 8.6 \mathrm{~mL}$ en el grupo A con cuantificaciones de $22.8 \mathrm{~mL} \pm 12.9 \mathrm{~mL}$ en el grupo B, encontrando diferencia estadísticamente significativa (Cuadro 2).

No surgieron complicaciones transoperatorias en ninguno de los grupos, las complicaciones postoperatorias observadas en el grupo A correspondieron a cinco pacientes (33.3\%); mientras que en el grupo B se presentaron en
20 casos $(62.5 \%)$, siendo la ventriculitis la más frecuente. De manera global en ambos grupos (Cuadro 2) se observó que la ventriculitis en todos los casos fue originada por Staphylococcus epidermidis que se resolvió con vancomicina.

Los días de estancia hospitalaria mostraron diferencia estadísticamente significativa teniendo $X$ de 15 días para el grupo A y de 26 días para el B (Cuadro 2).

No hubo fallecimientos en los siguientes 30 días al procedimiento en ninguno de los grupos.

En el seguimiento a un año de ambos grupos se requirió cambio valvular por disfunción, un caso en el grupo A y dos en el grupo B, sin diferencia estadísticamente significativa.

El estudio se dio por terminado el último día de diciembre de 2014. En virtud de los resultados antes descritos se decidió efectuar durante 2015 el procedimiento en un solo tiempo en todo paciente con mielomeningocele que cumpliera con los criterios de inclusión antes mencionados, conjuntando una serie de 26 pacientes adicionales.

Cuadro 2. Datos quirúrgicos de 47 pacientes con mielomeningocele con plastia y derivación ventrículo peritoneal en un solo tiempo quirúrgico (grupo A) y a la sumatoria de dos tiempos quirúrgicos separados en tiempo (grupo B).

\begin{tabular}{|c|c|c|c|c|c|}
\hline Variable & Grupo A & $\%$ & Grupo B & $\%$ & P. E. valor de $p$ \\
\hline \multicolumn{6}{|l|}{ T. Anestesia/min. } \\
\hline $\mathrm{N}$ & 15 & - & 32 & - & \\
\hline Mínimo & 90 & - & 105 & - & \\
\hline Máximo & 160 & - & 275 & - & $t=4.9$ \\
\hline X y DE & $111 \pm 22.1$ & - & $166 \pm 39.9$ & - & $p=0.00001 S$ \\
\hline \multicolumn{6}{|l|}{ T. Cirugía/min. } \\
\hline Mínimo & 60 & & 60 & & \\
\hline Máximo & 120 & & 205 & & $t=3.44$ \\
\hline X y DE & $81.7 \pm 19.5$ & - & $116 \pm 36.8$ & & $p=0.001 S$ \\
\hline \multicolumn{6}{|l|}{ Sangrado $\mathrm{mL}$} \\
\hline Mínimo & 3 & & 8 & & \\
\hline Máximo & 30 & & 55 & & $t=3.10$ \\
\hline X y DE & $11.4 \pm 8.6$ & & $22.8 \pm 12.9$ & & $p=0.003 S$ \\
\hline \multicolumn{6}{|l|}{ Complicac. P.O. } \\
\hline Ventriculitis & 2 & 13.3 & 6 & 18.7 & \\
\hline Dehiscencia HQ & 1 & 6.7 & 2 & 6.2 & \\
\hline Infección $\mathrm{HQ}$ & 0 & 0 & 6 & 18.7 & \\
\hline Neumonía intra $\mathrm{H}$. & 1 & 6.7 & 4 & 12.5 & \\
\hline Otras & 1 & 6.7 & 2 & 6.2 & P. exacta Fisher \\
\hline Total & 5 & 33.3 & 20 & 62.5 & $p=0.05 S$ \\
\hline \multicolumn{6}{|l|}{ Estancia hosp./días } \\
\hline Mínimo & 5 & & 7 & & \\
\hline Máximo & 48 & & 66 & & $t=2.53$ \\
\hline X y DE & $15 \pm 12.5$ & & $26 \pm 15.7$ & & $p=0.01 S$ \\
\hline
\end{tabular}

$\mathrm{N}=$ Número; $\mathrm{P} . \mathrm{E}$. = Prueba estadística; $\min .=$ Minutos; $\mathrm{NS}=$ No significativo; $\mathrm{S}=$ Significativo. $\mathrm{X}=$ Promedio; $\mathrm{DE}=$ Desviación estándar; $\mathrm{t}=\mathrm{t}$ de Student; P.O. = Postoperatorio. 


\section{DISCUSIÓN}

Es innegable que los avances tecnológicos así como la depuración de las técnicas quirúrgicas como la ventriculostomía ${ }^{15}$ endoscópica del tercer ventrículo (la cual presenta controversias, pero que en casos seleccionados de mielomeningocele puede llegar a evitar la necesidad de derivación ventricular y el manejo fetal del mielomeningocele ${ }^{16}$ que continúa en desarrollo) han permitido cambiar en gran medida su historia natural, ${ }^{17}$ del mismo modo han agregado nuevos capítulos a la historia de la hidrocefalia ${ }^{18}$ y sus múltiples complicaciones. No obstante, en nuestro medio aún es difícil adaptar dichos avances a la población general. La práctica de la plastia del meningocele y la colocación de derivación ventrículo-peritoneal en los pacientes que así lo requieren son técnicas quirúrgicas ampliamente conocidas. En las instituciones de salud pública en México el manejo del mielomeningocele y la colocación de sistemas de derivación ventrículo-peritoneal se efectúan en forma diferida; ${ }^{19}$ su realización en el mismo acto quirúrgico no es nuevo, como lo demuestran los estudios confrontados en que se conjuntan 270 procedimientos simultáneos, existiendo controversia en cuanto a resultados (Cuadro 3), ${ }^{20-30}$ pues en $64.4 \%(n=174)$ éstos son satisfactorios, concordantes con los obtenidos en el presente trabajo, no así en las series de Oktem et al. ${ }^{26}$ y de Arslan et al. ${ }^{28}$ en las que en 96 casos (35.6\%) reportan incremento de complicaciones, principalmente infección. En la serie de Oktem ${ }^{26}$ la incidencia de infección se duplicó en la cirugía simultánea, los gérmenes reportados con más frecuencia como causantes de la infección no difieren de lo encontrado en esta serie, siendo los principales Staphylococcus epidermidis y Staphylococcus aureus. Respecto al riesgo de infección en el sistema de derivación ventrículo-peritoneal, tanto Oktem ${ }^{26}$ como Arslan ${ }^{28}$ en Estados Unidos y Rome$\mathrm{ro}^{31}$ en México mencionan que los riesgos de infección se incrementan en el recién nacido por sistema inmunitario inmaduro, por tiempo quirúrgico mayor de 60 minutos y permanencia hospitalaria mayor de 10 días. La infección del LCR asociada a las derivaciones es una de las complicaciones más comunes que enfrentan los neurocirujanos. La incidencia por operación en las últimas series es de 4.2-6.2\% por paciente.

La muestra de los pacientes sometidos a un solo tiempo quirúrgico se obtuvo optando por el tercero de cada tres pacientes, de manera no probabilística, consecutiva y sistemática por conveniencia, ya que todos los casos del universo de trabajo mostraron características semejantes, por presencia de mielomeningocele así como de hidrocefalia catalogada como moderada que requeriría la derivación como el resto del universo estudiado. Por lo anterior consideramos que no existe sesgo en la toma de la muestra; sin embargo, estamos conscientes de que cuando la muestra es obtenida por conveniencia, suele existir sesgo sin posibilidad de extrapolar los resultados a toda la población. Otro aspecto relevante es que la adquisición de válvulas de derivación en la institución, por burocracia (desde el surgimiento del seguro popular) implica un proceso de licitación, lo que obliga a retraso (de 10 a 14 días) en la adquisición y posteriormente la

\begin{tabular}{lccc}
\multicolumn{2}{c}{ Cuadro 3. Series de pacientes en quienes se efectuó simultáneamente plastia de mielomeningocele y colocación de } \\
sistema de derivación ventrículo-peritoneal.
\end{tabular}


programación de la colocación de la misma, lo que significa mayor tiempo de hospitalización y retraso en el manejo correcto de estos pacientes.

En relación con la vía de abordaje abdominal para la colocación del catéter peritoneal en espacio de Grynfeltt existe la ventaja de que no se requiere cambiar de posición al paciente. Las ventajas de la cirugía simultánea son: que ofrece la oportunidad de mejoría en neonatos con función motora preservada, ${ }^{32}$ facilita la curación de la plastia del mielomeningocele y brinda protección por la derivación al tejido cerebral, evitando la dilatación ventricular progresiva. El hecho de reducir a un solo evento el riesgo anestésico como un solo tiempo quirúrgico repercute en acortamiento de la estancia hospitalaria, decremento en la morbilidad y complicaciones, lo que implica una considerable disminución del gasto hospitalario. No obstante lo anterior, existen al menos dos reportes en contra de su práctica por incremento de la presencia de infección.

Los resultados encontrados en este trabajo, pese a ser una serie pequeña, lograron modificar la conducta de tratamiento en la institución, por lo que cerrado el estudio todos los pacientes con mielomeningocele recibidos durante 2015 que cumplieron los criterios de inclusión ( $n=26)$ fueron sometidos a un solo tiempo quirúrgico, además de que se logró existieran al menos 2 válvulas de derivación en almacén de manera permanente, evitando retrasos en el manejo de estos casos.

\section{CONCLUSIÓN}

En esta serie la práctica de plastia del mielomeningocele y derivación ventrículo-peritoneal en un solo tiempo quirúrgico mostró ventajas en todos los aspectos, principalmente en la disminución de complicaciones.

\section{REFERENCIAS}

1. Saadai P, Farmer DL. Fetal surgery for myelomeningocele. Clin Perinatol. 2012; 39: 279-288.

2. Valdés HJ, Canún SS, Reyes PA, Navarrete HE. Mortalidad por defectos en el cierre del tubo neural en menores de 5 años de edad en México de 1998 a 2006. Salud Pública Méx. 2010; 52: 341-349.

3. Medina SA, Coutiño LB, Alvarado JG, Ramírez RJ. Epidemiología del mielomeningocele en niños menores de un año de edad en el Instituto Nacional de Pediatría. Rev Mex Med Phis Rehab. 2001; 13: 50-54.

4. Kshettry VR, Kelly ML, Rosenbaum BP, Seicean A, Hwang L, Weil RJ. Myelomeningocele: surgical trends and predictors of outcome in the United States, 1988-2010. J Neurosurg Pediatr. 2014;13:666678.

5. Adzick NS. Fetal surgery for spina bifida: past, present, future. Semin Pediatr Surg. 2013; 22:10-17.
6. Tamburrini G, Frassanito P, lakovaki K, Pignotti $F$ et al. Myelomeningocele: the management of the associated hydrocephalus. Childs Nerv Syst. 2013; 29:1569-1579.

7. Longoni M, Porcel J, Gerbaudo S. Mielomeningocele: epidemiología y relación con otras complicaciones neurológicas. Rev Col Med Fis Rehab. 2012;22: 117-122.

8. Marreiros $\mathrm{H}$, Loff $\mathrm{C}$, Calado $\mathrm{E}$. Who needs surgery for pediatric myelomeningocele? A retrospective study and literature review. J Spinal Cord Med. 2015;38:626-640.

9. Davis BE, Daley CM, Shurtleff DB, Duguay S, Seidel K, Loeser JD et al. Long-term survival of individuals with myelomeningocele. Pediatr Neurosurg. 2005; 41: 186-191.

10. Chern JJ, Muhleman M, Tubbs RS, Miller JH, Johnston JM, Wellons JC 3rd et al. Clinical evaluation and surveillance imaging in children with spina bifida aperta and shunt-treated hydrocephalus. J Neurosurg Pediatr. 2012; 9: 621-626.

11. Hirose S, Farmer DL. Fetal surgery for myelomeningocele. Clin Perinatol. 2009; 36: 431-438.

12. Pedreira DA, Zanon N, Nishikuni K, Moreira de Sá RA, Acacio GL, Chmait RH et al. Endoscopic surgery for the antenatal treatment of myelomeningocele: the cecam trial. Am J Obstet Gynecol. 2016; 214; 111 e1-111.e11.

13. Grynfeltt J. La hernie lombaire. Montpellier Med. 1866; 16: 329.

14. Lesshaft P. Die Lumbalgegend in Anat. Chirurgischer Hinsicht. Arch. f. Anat. u. Physiol. u. Wissensch. Med. Leipzig. 1870; 37: 264.

15. Beuriat PA, Szathmari A, Grassiot B, Plaisant F, Rousselle C, Mottolese $C$ et al. Role of endoscopic third ventriculostomy in the management of myelomeningocele-related hydrocephalus: a retrospective study in a single French Institution. World Neurosur. 2016; 87: 484-493.

16. Tulipan N, Wellons JC 3rd, Thom EA, Gupta N, Sutton LN, Burrows PK et al. Prenatal surgery for myelomeningocele and the need for cerebrospinal fluid shunt placement. J Neurosurg Pediatr. 2015; 16: 613-620.

17. Elgamal EA. Natural history of hydrocephalus in children with spinal open neural tube defect. Surg Neurol Int. 2012; 3: 112.

18. Aleksandar JM, Branka S, Radoj I, Meljnikov A. Hydrocephalus: history of surgical treatment over the centuries. Istorija Medicine. 2012; 7: 119-125.

19. Guía de Práctica Clínica. Prevención, diagnóstico y tratamiento de la espina bífida en niños. México. Instituto Mexicano del Seguro Social 2013.www. CENETEC salud.mx/interior/gpc.html.

20. Epstein NE, Rosenthal AD, Zito J, Osipoff M. Shunt placement and myelomeningocele repair: simultaneous vs sequential shunting. Review of 12 cases. Childs Nerv Syst. 1985; 1: 145-147.

21. Hubballah MY, Hoffman HJ. Early repair of myelomeningocele and simultaneous insertion of ventriculoperitoneal shunt: technique and results. Neurosurgery. 1987; 20: 21-23.

22. Bell WO, Arbit E, Fraser RA. One-stage meningomyelocele closure and ventriculoperitoneal shunt placement. Surg Neurol. 1987; 27: 233-236.

23. Chadduck WM, Reding DL. Experience with simultaneous ventriculoperitoneal shunt placement and myelomeningocele repair. J Pediatr Surg. 1988; 23: 913-916.

24. Miller PD, Pollack IF, Pang D, Albright AL. Comparison of simultaneous versus delayed ventriculoperitoneal shunt insertion in children undergoing myelomeningocele repair. J Child Neurol. 1996; 11: 370-372.

25. Machado HR, Santos de Oliveira R. Simultaneous repair of myelomeningocele and shunt insertion. Child's Nerv Syst. 2004; 20: 107-109.

26. Oktem IS, Menkü A, Ozdemir A. When should ventriculoperitoneal shunt placement be performed in cases with myelomeningocele and hydrocephalus? Turk Neurosurg. 2008; 18: 387-391. 
27. Radmanesh F, Nejat F, El Khashab M, Ghodsi SM, Ardebili HE. Shunt complications in children with myelomeningocele: effect of timing of shunt placement. Clinical article. J Neurosurg Pediatr. 2009; 3: 516-520.

28. Arslan M, Eseoglu M, Gudu BO, Demir I, Kozan A, Gokalp A et al. Comparison of simultaneous shunting to delayed shunting in infants with myelomeningocele in terms of shunt infection rate. Turk Neurosurg. 2011; 21: 397-402.

29. Sinha SK, Dhua A, Mathur MK, Singh S, Modi M, Ratan SK. Neural tube defect repair and ventriculoperitoneal shunting: indications and outcome. J Neonatal Surg. 2012; 1: 21-24.
30. Akgun B, Ucler N, Erol FS, Kaplan M, Gediz T, Yilmaz I. The timing of ventriculo-peritoneal shunt application at the late stage myelomeningocele repair. Firat Tıp Dergisi. 2012; 17: 6-9.

31. Romero GM, León RA, Carreón GJ, Romero GF. Factores de riesgo de infección del sistema de derivación ventriculoperitoneal en pacientes pediátricos. Enf Inf Microbio. 2013; 34: 59-63.

32. Arredondo-Navarro LA, Soto MJ, González-González ME, AguirreJauregui O, Soto-Blanquel JL, Angulo CE y cols. Importancia del manejo temprano del mielomeningocele. Estudio comparativo. Rev Med MD. 2013; 4: 73-77. 